\title{
Rituels contemporains de première menstruation
}

\author{
Michael Houseman, \\ CEMAF/EPHE*
}

L'invention de nouvelles formes cérémonielles - liées au mariage [Segalen, 2003] aux funérailles [Biot, 1993], aux commémorations nationales [Handelman, 2004], aux mouvances New Age et néopaïens [Galinier et Molinié, 2006] - est monnaie courante [voir aussi Grimes, 2000 ; Fellous, 2001 ; Dianteill et al., 2004]. Quel statut faut-il reconnaître à ces innovations, souvent marginales ou issues d'initiatives individuelles? Doit-on les traiter comme des actions rituelles à part entière, ou obéissent-elles à des principes d'organisation qui marquent une rupture radicale avec les activités ritualisées que l'on peut observer dans d'autres traditions? Cette question se pose avec d'autant plus d'acuité lorsque les pratiques en question sont explicitement désignées par les participants comme des « rites de passage » destinés à médiatiser l'accession d'une jeune personne au statut d'adulte. C'est le cas des événements cérémoniels que l'on se propose de considérer ici : des rites célébrés à l'occasion des premières règles.

Ces rituels, appelés "célébrations de la première lune », "cérémonies du premier sang ", "rites de ménarche", "fêtes rouges ", etc., documentés dans quelques ouvrages [Fellous, 2001 ; Salomonsen, 2002], sont décrits principalement sur Internet, dans des forums de discussion, des blogs, et surtout sur des sites consacrés à la menstruation, au Néopaganisme et à la spiritualité féminine. Il s'agit pour l'essentiel de témoignages personnels, de canevas rituels fournis par des individus ou collectifs, et de composants de «kits» cérémoniels vendus sur des sites commerciaux (Figure 1).

Tous s'attachent, de près ou de loin, au Néopaganisme, lequel a émergé en Angleterre dans les années 1930, pour devenir un mouvement plus minoritaire que marginale dans le paysage religieux des Etats-Unis [voir par exemple LassalletteCarassou, 2008]. Les Néopaïens (ou Païens), qui se répartissent en divers courants (Wicca, Druidisme, etc.), cherchent, par leurs pratiques cérémonielles, à renouer avec une sensibilité préindustrielle fondée sur le corps, les rythmes de la nature et l'interconnexion profonde des êtres et des choses. Leurs théogonies diffèrent d'un courant à l'autre, mais aménagent une place importante à la figure de la Déesse, ellemême souvent associée à la Terre(-Mère).

Certaines de ces cérémonies sont d'ordre familial, rassemblant une jeune femme, ses amies, sa mère et des proches de celle-ci, tandis que $d$ 'autres s'apparentent plus à des festivités communautaires [cf. Houseman, 2007]. Les descriptions qui suivent concernent un rite célébré au sein de la communauté néopaïenne d'une grande ville, un autre impliquant les membres d'une famille et leurs amis, et une série de trois variantes s'inscrivant plus directement dans la tradition Wicca et rassemblant des individus d'un même coven (cellule de cocélébrants).

\footnotetext{
* Paru dans Ethnologie française 40 (1) : 57-66, 2010 (« Nouvelles Adolescences »)
} 


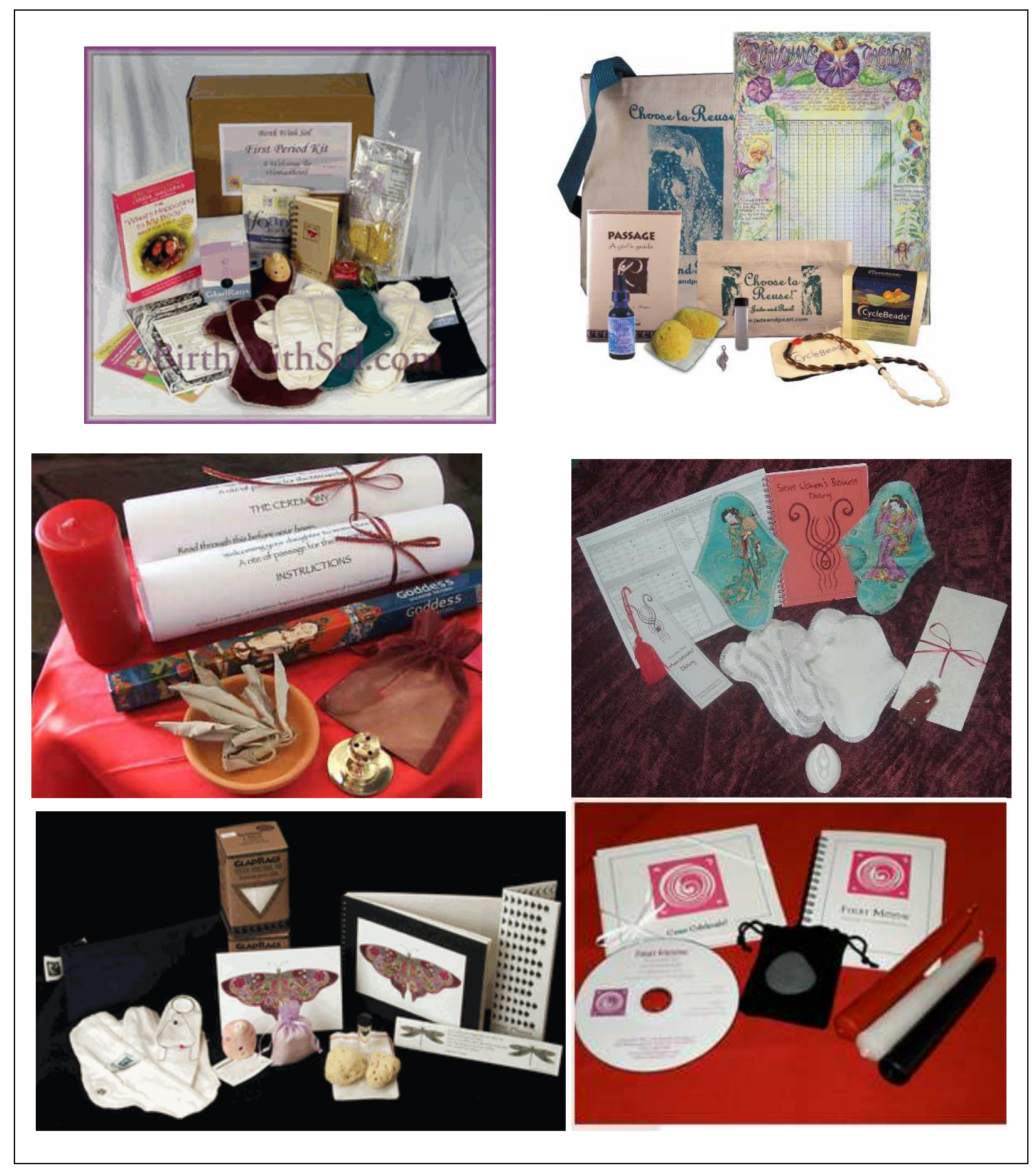

Ill. 1 Quelques « kits » vendus sur l'Internet pour la célébration des premières règles

\section{Un rituel pour Sonia}

Cette cérémonie, rapporté par Salomonsen [2002], a eu lieu en 1989 à San Francisco. Présidé par Starhawk, une des figures les plus influentes du mouvement néopaïen, elle a été célébrée pour Sonia, jeune femme élevée comme néopaïenne. Lorsque Sonia a eu 13 ans, Anna, sa mère, lui demanda de se choisir un nouveau 
nom rituel et de préparer quelque chose de personnel qu'elle aura à offrir à la communauté. Lorsque Sonia a ses premières règles quelques mois plus tard, mère et fille discutent du rite à venir, s'accordant sur onze autres participants. L'organisation de la cérémonie sera en grande partie assurée par Hera, la mère de Nicole qui est une amie de Sonia et dont les premières menstruations ont été célébrées quelques années auparavant.

Le jour de la célébration, les femmes, habillées dans leurs habits rituels, se rendent dans un grand parc public. Elles forment un cercle autour de Sonia et Starhawk verse de l'eau sur le front, les seins, le ventre et le sexe de la jeune femme, déclarant «Souviens toi : personne ne peut te donner du pouvoir. $\mathrm{Tu}$ as déjà ce pouvoir en toi ».

Le poignet droit de Sonia et le poignet gauche de sa mère sont ensuite reliés par une corde. Hera explique à la postulante : "Lorsque tu es née, tu es venue au monde liée au corps de ta mère. Tout comme le cordon ombilical a dû être coupé à ce moment-là pour que tu puisses vivre, de la même façon, cette corde entre vous deux doit être coupée. Mais le lien entre vous ne sera jamais coupé, car c'est un lien du cœur ». Starhawk demande alors à mère et fille de courir ensemble dans le parc tant qu'elles le peuvent. Quelque temps après, la mère fait savoir qu'elle n'arrive plus à maintenir le rythme. Lorsqu'on les fait revenir dans le cercle, la grand-mère de Sonia coupe la corde qui la relie à sa mère à l'aide d'un couteau cérémoniel.

Sonia doit ensuite courir seule à travers un bois jusqu'à la statue de Diane la chasseresse pour y méditer «sur Diane, la déesse vierge, et sur ta propre arrivée à l'état de jeune femme ». Sonia s'exécute et les autres femmes suivent à pas de marche, portant des fleurs dans les bras. Lorsqu'elles arrivent à la statue, elles forment un cercle à côté de Sonia. Nicole rassemble les fleurs dans un grand panier qu'elle donne ensuite à Sonia puis, en prenant la main de celle-ci, l'amène au centre du cercle. Les femmes se tiennent serrées les unes contre les autres et lorsque Starhawk commence à taper de son tambour, les femmes «apprennent à Sonia ce qu'est vraiment la Déesse » en répétant en chantent: «Ecoute, écoute ma chanson de cœur ; je ne t'oublierai jamais, je ne $t^{\prime}$ abandonnerai jamais; je t'aimerai toujours, je serai toujours avec toi ». Pendant un long moment, Sonia, intimidée, rougit et regarde ses pieds. Alors que le chant se poursuit, elle lève la tête et regarde les femmes à tour de rôle calmement dans les yeux. Toutes les participantes paraissent très émues et certaines, dont Anna, la mère, commencent à pleurer, tout en continuant à chanter.

La seconde étape a lieu chez Anna. Les femmes, assises en cercle, racontent à Sonia des histoires personnelles sur leurs menstruations: les circonstances de leurs premières règles, les réactions de leurs partenaires, des conseils, le potentiel magique des menstrues, etc. Sonia révèle ensuite son nouveau nom à l'assemblée: Aurora. Mère et fille sont placées au centre du cercle et les femmes leur chantent: "C'est le sang des Anciens qui coule dans nos veines / les formes passent mais le cercle de la vie demeure ». Les femmes entreprennent de produire une énergie mystique (un "cône de pouvoir») qu'elles « ancrent » dans Sonia/Aurora. Enfin, elles lui offrent des cadeaux : 
sous-vêtements en soie, figures de la Déesse, une ceinture, des préservatifs, etc., ainsi que le couteau cérémoniel utilisé pour couper la corde entre sa mère et elle.

La dernière phase du rituel a lieu en début de soirée dans une salle d'un local municipal. Richard, le père de Sonia, a aménagé la salle avec quatre autels pour les quatre éléments (vent, feu, eau, terre) et une table où se trouve un festin avec une majorité de plats de couleur rouge (saumon, mousse à la fraise, etc.) et un gâteau sur lequel figure le nom " Aurora ». Une soixantaine d'invités sont présents.

Richard souhaite la bienvenue à sa fille, à la fois en tant que Sonia et en tant qu'Aurora. Les participants créent un cercle et convoquent la Déesse. Ils font circuler un petit bocal de perles d'où les hommes prélèvent une perle rouge et les femmes une perle blanche ; une fois qu'ils les ont « chargées » de bonnes intentions en leur soufflant dessus, les perles sont remises à Sonia qui doit en faire un collier comme signe de l'amour que lui porte la communauté. Ensuite, c'est à Sonia d'offrir son cadeau à la communauté : elle exécute une danse de ballet classique dans une robe blanche au centre du cercle. L'assemblée entreprend alors une "danse spirale», et lorsque l'énergie engendrée est au plus fort, les femmes se précipitent au centre du cercle, soulèvent Sonia et la balancent au bout de leurs bras. Une autre séance de dons offerts à Sonia achève le rituel, et la soirée se prolonge avec de la musique rock, des danses et le festin.

\section{Un rituel pour Tisa}

Après avoir consulté l'Internet, des ouvrages spécialisés et diverses connaissances, Ksenija Soster-Olmer [2001] a organisé une «Fête Rouge » pour sa fille aînée, Tisa. Cette dernière avait elle aussi des idées sur la question : "Maman, peut-on avoir beaucoup de bougies? Ou un feu ? Et des couronnes de fleurs ? J'ai vu cette façon géniale d'arranger les cheveux comme faisaient les Grecs d'autrefois ». Ayant décidé avec sa fille des personnes à inviter, la mère demande à toutes les participantes d'apporter avec elles une pierre, et de préparer une bénédiction qu'elles auraient à prononcer. Elle demande à Tisa d'y apporter quelque chose à laquelle elle tient et qui représente pour elle son enfance, en la prévenant qu'elle sera obligée de l'abandonner. Tisa choisit son animal en peluche le plus ancien.

Les participantes, cinq filles et sept femmes dont trois sont des mères des filles présentes, se rassemblent en début de soirée dans la maison de la mère de Tisa. Toutes portent avec ostentation quelque chose de couleur rouge ; Tisa est elle-même habillée dans la robe de mariage teinte en rouge de sa mère. Des pétales de rose dessinent un passage entre la porte d'entrée et l'autel situé au centre du salon. L'autel est composé d'un tissu rouge posé par terre sur lequel ont été placés, aux points cardinaux, des objets représentant les quatre éléments : des plumes pour l'air (est), une bougie et de l'encens pour le feu (sud), un bol d'eau de mer et de l'huile pour l'eau (ouest) et un bol d'argile rouge pour la terre (nord). Au centre de l'autel est posé entre deux bougies blanches une couronne de marguerites que la mère a préparée pour sa fille. 
Les membres de l'assemblée se mettent debout autour de l'autel, chaque fille tenant une bougie rouge (éteinte) à la main. L'officiante remercie les quatre éléments de leur présence et parle de ce qu'ils signifient dans la vie d'une femme (l'air représente la pensée, les idées et la sagesse féminine ; le feu, les passions et la sensualité, etc.). En se tenant par les mains, l'assistance chante « une belle chanson amérindienne : Elle coule, la rivière, elle grandit ; elle se déverse dans la mer. Porte moi, ma mère, je serai toujours ton enfant. Porte moi, ma mère, jusqu'à la mer. Elle coule, la rivière ».

La mère présente à sa fille une boîte enveloppée de papier rouge. A l'intérieur, sous de nombreuses couches de papier de soie, se trouve une boîte plus petite entourée d'un ruban de velours rouge, qui contient une petite statuette en argile de la "Déesse de la Menstruation» (Figure 2). La mère explique à l'assistance: "Tout comme Tisa a dû traverser ces multiples enveloppes pour atteindre la petite statuette, je souhaite que chacune d'entre nous descende jusqu'au plus profond d'elle-même pour partager ses pensées avec elle ». En faisant circuler la statuette, les participantes parlent des femmes qu'elles admirent, ainsi que de leurs mères et grand-mères, évoquant «les difficultés et les joies de devenir et d'être une femme ».

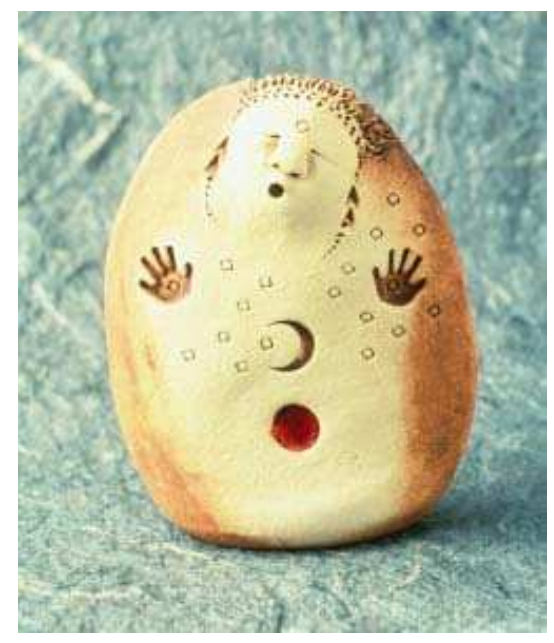

Ill. 2 Déesse menstruelle de $5 \mathrm{~cm}$ du Bell Pine art farm [www.bellpineartfarm.com] vendue sur le site www.birthwithsol.com : "remplir ta Déesse avec une goutte de sang menstruel et sceller avec de la cire de bougie »

Ensuite a lieu «la cérémonie du seuil ». Le ruban en velours rouge est placé parterre de manière à diviser les participantes en deux groupes : d'un côté, la mère de Tisa et les autres femmes, de l'autre, les filles, dont Tisa, la couronne de marguerites sur la tête et son animal en peluche dans les bras. On allume les bougies rouges que portent les autres filles; celle que tient Tisa reste éteinte. A la question «Qui s'approche du seuil ? ", Tisa donne son nom. Puis, lorsqu'on lui demande "Es-tu prête à abandonner derrière toi ton enfance en devenant une jeune femme ? », elle répond en chuchotant : « Non ». La mère intervient: "Alors, lorsque tu seras prête à te joindre à notre cercle, laisse ton jouet derrière toi ». Tisa ferme les yeux, respire profondément et 
dépose son jouet parterre ; elle enjambe le ruban rouge et, en pleurant, tombe dans les bras de sa mère.

Sa mère l'accueille en disant : "Bienvenue, jeune femme [Maiden] sur la voie qui te mène à devenir une femme ». Elle utilise les objets de l'autel pour bénir sa fille: elle touche Tisa avec les plumes («que le vent remplisse tes voiles et te fasse $t^{\prime}$ envoler»), fait tourner la bougie autour d'elle («que le feu ne te brûle pas, mais te rende forte »), etc. La mère lui offre ensuite une cuillerée de miel ("Grandir apporte avec lui la douceur de la vie...»), et lui met du sel sur la langue («...ainsi que l'amertume de la vie...»). Elle lui donne aussi une grosse fraise rouge à manger ( $Q$ Que tu n'aies jamais faim ») et $\mathrm{du}$ jus de groseille à boire («Que tu n'aies jamais soif»). Enfin, elle lui présente un collier en forme de cœur ("Que ce cœur te protège») et un bracelet de coquillages («que ce bracelet t'enlace dans l'amour de ta famille»). Les filles, restées de l'autre côté du ruban, se penchent alors pour allumer la bougie rouge de Tisa avec les leurs.

Contemplant l'assemblée souriante et en pleurs, la mère de Tisa lui dit: «Lorsque tu rencontreras des peurs et des difficultés sur ta route de femme, rappelle-toi que tu n'es pas seule. Voici ton cercle de femmes qui t'aideront et te guideront. Et souviens-toi que je serai toujours là pour toi, quelle que soit la lourdeur de ton fardeau ». Elle remet ensuite à Tisa l'animal en peluche que celle-ci a abandonné, en lui expliquant: "Bien que tu sois maintenant une jeune femme, il est toujours bon de savoir que tu as une précieuse petite fille à l'intérieur de toi. Garde ceci comme un symbole de ton enfance et de mon amour pour toi ».

A tour de rôle, les autres participantes présentent à Tisa les pierres qu'elles lui ont apportées, en énonçant leurs bénédictions. Tisa place toutes ces pierres dans un tissu noir qu'elle noue avec le ruban de velours rouge. Le rituel se termine alors avec la récitation d'un « poème de puberté Navajo » et un festin de nourritures rouges: vin et jus d'airelles, pâtes avec poivrons rouges, risotto avec tomates, mousse de framboise, pastèque, etc.

\section{Trois variantes wiccan}

Les trois « rites de passage » suivants, célébrés dans le cadre d'une réunion de coven (mixte), sont dirigés par une Grande-prêtresse et comportent une rencontre entre la postulante et des participants qui incarnent les Quartiers ou points cardinaux d'un cercle rituel.

Le script du premier a été écrit par Arwen Nightstar pour son grimoire en ligne [www.anisoptera.com/grimoire/comeage.htm]. La jeune femme, habillée dans une robe blanche ceintrée d'un cordon rouge, est présentée aux quatre Quartiers. Chacun lui fait don de qualités spécifiques et d'un objet emblématique: l'Est, qui représente «le vent, l'air, le souffle même que tu respires » et qui apporte à la postulante « intelligence et esprit », lui donne une plume ou un éventail ; le Sud, qui représente « le feu, la flamme, la chaleur qui chauffe ton sang » et lui apporte " passion et amour ", lui offre une bougie, etc. A chaque fois, en réponse à la question «Et que m'apporteras-tu ? », la jeune 
femme abandonne un de ses jouets préférés. Elle est ensuite conduite par la Grande-prêtresse à l'autel où attend Esprit : « Je suis Esprit, le centre, le noyau, l'amande de la vie qui est unique en toi. Je t'apporterai ton identité psychique. Je t'apporterai la Déesse et le Dieu. Voici un don librement donné : une image de la Déesse, elle qui a mille noms, elle qui est ». Esprit remet à la postulante un miroir recouvert d'un tissu, et lorsque la jeune femme s'y regarde, lui explique : «Tu es la Déesse. La Déesse est toi. Que tu te traites avec amour et respect et honneur car la Dame ne mérite pas moins». La jeune femme remercie Esprit et offre en retour son jouet le plus cher, don qui «symbolise mon dernier lien avec l'enfance et ma volonté de devenir une adulte ». Après avoir fait circuler ce jouet parmi les membres de l'assemblée, chacun formulant un souhait à l'intention de la postulante, Esprit le soulève à bout de bras: "Ton cadeau est pour la Déesse et à la Déesse il doit revenir ». En rendant le jouet à la postulante, Esprit continue : «Bien que tu ne sois plus une fille, garde ceci pour te souvenir que Tu es Déesse. Ne permets jamais à l'enfance de s'éloigner de toi au point où tu ne pourrais plus rire aisément ou participer à un jeu sans conséquence ». S'adressant alors à l'assemblée, Esprit déclame : «Je vous présente [le nom de la postulante], une femme ».

Le rituel suivant a été écrit en 2002 par les membres de l'Ordre Sybiline [www.sibyllineorder.org/rituals/rit_passagerite_woman.htm]. La jeune femme, habillée en blanc et ayant allumé une bougie rose («qui représente ton enfance » lui explique-t-on) sur l'autel, entreprend un tour des Quartiers. Comme dans le cas précédent, elle reçoit des dons de chacun, ainsi qu'un miroir de la part d'Esprit (avec des paroles similaires), mais ne leur remet rien en retour. Elle dépose ensuite le miroir sur l'autel et revient avec un cordon qu'elle a précédemment tressé à partir de rubans de différentes couleurs. Sa mère la rejoint au centre du cercle et la Grande-prêtresse tend la corde entre mère et fille, en expliquant qu'elle "représente son ultime pas pour devenir femme car, tout comme le cordon ombilical maternel lui a donné de la nourriture pour la vie, ce cordon représente des attaches l'associant aux flux et aux reflux de la vie». La jeune femme prend le cordon, et (sans aide extérieure, précise le rite) le noue autour de sa taille. La Grande-prêtresse poursuit : "Aucune femme de notre communauté ne reste seule; il en va de même pour cette jeune femme. Car, en quittant la protection de sa mère, elle s'est tressé une corde qui la relie au monde autour d'elle». La postulante allume alors une bougie rouge posée sur l'autel, et la Grande-prêtresse déclare à l'assemblée «Je vous présente [le nom de la postulante] qui n'est plus une enfant mais une femme avec les droits et les responsabilités d'une femme ».

Le dernier rite a été créé par Eustacia Blackstar en 2001 pour sa fille [www.moonpathcuups.org/rituals/coa.htm]. La jeune femme, après avoir énoncé le nouveau nom qu'elle s'est choisi (Perséphone), va à la rencontre des "Quatre vents » (Esprit n'est pas mentionné). Comme dans le cas précédent, elle reçoit de chacun d'eux des cadeaux sans rien offrir en retour. La Grandeprêtresse (qui est aussi la mère de la postulante) offre aussi un cadeau à la jeune femme, en disant "Ce témoignage de mon amour pour toi est pour symboliser le lien éternel entre mère et enfant». Ensuite, l'ensemble des 
femmes, convoquées par la Grande-prêtresse en tant que mères actuelles ou potentielles, forment deux rangées qui font face et, élevant leurs bras, composent ensemble " une représentation symbolique de la voie de sortie du bébé (birth canal) ». La Grande-prêtresse demande au Grand-prêtre de relier mère et fille "une dernière fois » avec un cordon argenté, puis la fille, suivie de sa mère, passe sous les bras des femmes. Arrivée au bout de la "voie de sortie du bébé", la jeune femme lève les bras ("dans la position de la Déesse ») et le cordon qui la relie à sa mère est coupé. On allume les bougies placées sur un gâteau lui-même posé sur l'autel et la Grande-prêtresse demande à la postulante «comme premier acte de magie» de souffler les bougies afin « d'envoyer tes rêves dans l'univers ». Enfin, comme son premier devoir sur son "nouveau chemin», la jeune femme clôt la séance en remerciant la Déesse et en renvoyant les Quartiers.

\section{Les enjeux des rites de première menstruation}

Judith Brown [1963] constate, à partir d'un échantillon de 75 sociétés, une forte corrélation entre la présence de rites d'initiation féminine et le fait que les filles, une fois mariées, continuent à résider là où elles ont grandi, lieu où réside aussi leur mère. Une continuité résidentielle ainsi qu' une proximité soutenue entre mère et fille, favoriserait la mise en place de dispositifs initiatiques marquant, pour la fille comme pour son entourage, à la fois son autonomie par rapport à sa mère et l'accession à ses nouveaux droits et devoirs d'adulte.

Les enjeux des rites de passage féminins que met en évidence cette étude ne sont pas étrangers à ceux qu'on peut être tenté d'attribuer aux rituels des premières règles. Ces derniers intègrent bon nombre d'éléments qui mettent en scène une rupture entre la jeune femme et son état d'enfant, coupure qui est ensuite consacrée par une incorporation à la collectivité des femmes adultes : exclusion du cercle des femmes puis intégration à celui-ci, le fait d'enjamber une ligne séparant les filles des femmes, ou de traverser une "voie de sortie du bébé ", l'abandon de jouets, l'adoption d'un nouveau nom, etc. Et dans bien des cas, comme en témoigne la coupure du cordon reliant mère et fille ou la récupération définitive du cordon par cette dernière, cette rupture concerne plus particulièrement le lien entre la jeune femme et sa mère.

Toutefois, en centrant l'attention sur des finalités que peuvent suggérer un rapprochement rapide avec des institutions initiatiques dans d'autres cultures, on risque de négliger non seulement les particularités des performances rituelles, mais aussi la spécificité du «travail » de la ritualisation qui y est à l'oeuvre. Il est, par exemple, difficile de soutenir que la transformation que médiatisent les rites qui nous concernent consiste en un "passage » du statut d'enfant à celui d'adulte. L'ensemble des personnes impliquées, dont en premier lieu la jeune femme elle-même, sont conscientes que celle-ci occupera pendant longtemps encore une position de mineure au sein de sa famille comme dans la société. Disons plutôt qu'à l'instar de tous les rituels, ces célébrations mettent en place une nouvelle définition de la postulante qui, bien qu'incontestablement expérimentée par les participantes, demeure difficilement intelligible en dehors de la performance qui l'actualise [Houseman 2006]. C'est en se 
penchant sur l'originalité de ces rituels qu'on sera plus à même de saisir la nature de cette redéfinition.

La célébration des premières règles instaure une configuration quelque peu paradoxale dans laquelle le lien à l'enfance et à la mère est à la fois coupé de façon ostensible et soigneusement préservé : les jouets abandonnés par la jeune femme lui sont restitués, et sur le plan discursif on insiste sur le besoin qu'a la postulante de rester une précieuse petite fille à l'intérieur d'elle-même, et de ne jamais permettre à l'enfance de s'éloigner d'elle; de même, on accentue la pérennité du lien mère-fille qui ne sera jamais coupé, car " $c^{\prime}$ est un lien du cœur». Ainsi, tout en renonçant à l'enfance, la jeune femme est requise de garder, à la fois « à l'intérieur » d'elle-même et sous la forme de représentations matérielles qu'elle est censée conserver - le jouet qui lui est rendu, le couteau qui a servi à couper le cordon, le cadeau-témoignage de l'amour maternel - l'expérience de cette enfance abandonnée.

Ce dédoublement interne de la jeune femme - elle porte désormais en elle la petite fille qu'elle était - a comme pendant un rapprochement réflexif où la postulante est encore une fois dédoublée au moyen de son identification à la Déesse. Le don par Esprit du miroir dans lequel la jeune femme découvre sa véritable nature est explicite à cet égard. Mais cette identification se retrouve aussi dans l'attribution de nouveaux noms (Aurora, Perséphone), dans la « position de la Déesse » qu'adopte la jeune femme, et dans le jeu de regards réverbérant entre la postulante et le cercle des femmes qui «apprennent à Sonia ce qu'est vraiment la Déesse » en chantant de façon répétitive « je t'aimerai toujours, je serai toujours avec toi ». Cette amplification supplémentaire de la postulante renvoie également à la fois à sa personne, au travers d'une nouvelle image d'elle-même, et aux objets concrets qui lui sont remis : miroir, fleurs, bijoux, statuettes, etc. Dans un des récits, ces deux mouvements de dédoublement - l'un vers intérieur, l'autre vers l'extérieur - se rejoignent expressément lorsque le jouet associé à son enfance qu'offre la jeune fille à la Déesse lui est restitué : «Ton cadeau est pour la Déesse et à la Déesse il doit revenir ».

Dire que ces rituels permettent à une jeune fille de devenir adulte est donc pure glose qui ne restitue pas la spécificité de ces événements. Il serait plus juste de dire qu'ils permettent à la jeune fille, comme aux autres participantes, d'expérimenter la nouvelle identité de femme qui y est actualisée : celle d'une Déesse (la postulante elle-même) qui porte en elle un enfant (la postulante elle-même).

Comment doit-on comprendre cette identité féminine? La quasi-totalité des rites de puberté destinés aux filles dans d'autres contextes culturels, sont explicitement rattachés à leur capacité nouvellement acquise de procréer ${ }^{1}$; ils font souvent partie intégrante des cérémonies préalables au mariage. De ce point de vue on ne peut qu'être frappé par le peu d'éléments faisant référence à la reproduction dans ces rites contemporains. Le cordon ombilical nourricier reliant la jeune femme à sa mère est évoqué, soit pour être symboliquement coupé, soit pour représenter les

\footnotetext{
${ }^{1}$ Un exemple européen est la gifle (suivie par une étreinte affectueuse) que donne une mère à sa fille lorsque celle-ci lui confie l'arrivée de ses premières règles. Acte de transmission où sont condensés des modes de relation antithétiques, cet épisode, que l'on retrouve par exemple dans le film Diabolo menthe de Diane Kurys (1977), s'imposera à celles qui le subissent comme pierre de touche évocatrice mais particulièrement résistante à l'analyse pour des comportements et discours postulant un lien étroit entre féminité et maternité [Houseman, 2007].
} 
« attaches aux flux et aux reflux de la vie » qui relient la jeune femme " au monde autour d'elle». Les histoires personnelles que partagent les femmes avec la postulante ne font pas mention d'accouchement ou d'enfants, mais restent centrées sur le phénomène de la menstruation : conseils pratiques, réactions de l'entourage, significations ésotériques des menstrues, etc. Et si la couleur rouge est partout présente - dans les vêtements, les objets cérémoniels, les nourritures, etc. - elle se rapporte non pas à une métaphore des capacités génésiques, mais entretient un rapport métonymique avec les pertes sanguines. Cette minoration rituelle du processus procréateur rejoint l'argument de M. Moisseeff [2004 et dans ce numéro] pour qui la responsabilité parentale dans l'Occident contemporain, contrairement à ce qu' on peut observer dans d'autres cultures, consiste non à légitimer leurs enfants à user de leur fonction procréative mais à leur fournir les moyens de s'en prémunir.

Ces rituels sous-tendent non l'accès à la procréation, mais à la création, ou plus exactement, à la créativité « spirituelle ». En favorisant l'éclosion de « ce pouvoir en toi » de la postulante, en faisant d'elle l'objet de bénédictions, en la situant comme point de mire des énergies soulevées par l'assistance, en médiatisant sa rencontre avec les puissances associées aux points cardinaux, en promouvant sa confrontation réflexive avec la Déesse, ces rituels opéreraient un réveil spirituel chez la jeune femme qui les subit. En actualisant chez elle une identité féminine doublement augmentée, c'est-à-dire en faisant d'elle un être spirituel (la Déesse) recélant une vitalité inventive (l'enfance), les rites des premières règles la consacrent en tant qu'agent indépendant doté de capacités de création. Ils font d'elle une personne féminine à part entière à même, par exemple dans le dernier récit, d'assumer les droits et devoirs cérémoniaux qui incombent aux membres adultes du coven.

On retrouve ici une conception recomposée de l'identité féminine qui confère à la menstruation une place centrale. Véhiculée notamment par les écrits de Lara Owen [1993] et par des mouvements s'inspirant de La tente rouge d'Anna Diamant [1997], elle présente la féminité sous trois aspects : une figure maternelle protectrice, une figure de femme «sauvage » (comme la déesse Diane) ne connaissant ni rapports sexuels ni procréation, et une figure de la femme-amante passionnée, équi-statutaire avec l'homme et chez qui sexualité et spiritualité sont étroitement conjuguées [cf. Fedele, 2008]. Propriété exclusive de la femme-amante, le sang menstruel n'intervient plus comme le signe d'un pouvoir procréateur potentiel ou avorté, mais comme l'indice d'une compétence spirituelle constitutive de l'état de femme. Par le biais de leurs cycles menstruels, ainsi qu'au travers des échanges qu'ils permettent avec d'autres femmes, et éventuellement, avec la Terre-mère elle-même, les femmes participeraient aux mouvements génératifs qui régissent le devenir du monde.

L'identité adulte à laquelle donnent accès ces rituels ne renvoie donc ni à un statut social particulier, ni à une qualité de procréatrice potentielle, mais à la reconnaissance d'une créativité spirituelle. Dans de très nombreuses sociétés où le statut de femme adulte est étroitement associé à la maternité, et où la capacité pour une femme de devenir mère est en partie fonction du lien avec sa propre mère, la transmission des pouvoirs génésiques qui médiatise son accession au statut d'adulte, en ce qu'elle comporte implicitement ou explicitement une cessation des activités procréatives chez la mère, est conçue comme potentiellement problématique [par exemple Héritier, 1977]. Ainsi, ce sont généralement d'autres femmes qui 
interviennent afin de médiatiser ce passage. Or, la situation se présente tout autrement ici : ce sont typiquement les mères qui organisent les rites des premières règles pour leurs filles. L'idée d'une rivalité entre mère et fille n'est pas totalement absente de ces célébrations, comme en témoigne la course à pied entre Sonia et sa mère. Mais de façon générale, la mère y intervient comme représentante protectrice et interlocutrice privilégiée pour sa fille au sein de la communauté des femmes qu'elle rejoint. C'est que l'accession rituelle de la jeune femme à une autonomie créative n'enlève rien à cette même qualité chez sa mère. Bien au contraire, faire parvenir sa fille à l'état d'adulte spirituel non seulement renforce leurs liens en tant que membres d'une communauté féminine solidaire, mais participe à la promotion de la mère au sein de cette communauté. Lorsque la mère aura atteint la ménopause, elle sera consacrée Crone (Vielle sage), statut hautement valorisé qui comportent des responsabilités accrues. De ce point de vue, les rites des première règles concernent autant les mères que les filles elles-mêmes, faisant d'elles des partenaires, situées à des stades d'avancement différents, dans la construction d'une identité féminine commune.

\section{Des rites comme les autres?}

A l'instar d'autres événements cérémoniels de par le monde, les rites contemporains des premières règles ont indubitablement un sens : ils transmettent un certain nombre d'idées et de valeurs. On peut également leur reconnaître des fonctions ou conséquences pragmatiques, autant psychologiques que sociales. Toutefois, ce qui fait d'une séquence d'actes un rituel ne réside ni dans le symbolisme qui y serait à l'œuvre (lequel est commun aux autres aspects de la culture), ni dans les fonctions qu'on peut lui attribuer (qui, en soi, n'exigent nullement un recours à des dispositifs cérémoniels). L'efficacité caractéristique des pratiques rituelles, qui les distinguent, par exemple, des interactions quotidiennes et du jeu ou du spectacle, se rapporte plutôt aux principes qui président à l'organisation de la performance ellemême. Or, sur ce plan, ces célébrations s'écartent nettement des événements que les ethnologues ont $l^{\prime}$ habitude de désigner comme « rituels ».

Tout d'abord, et sans que cela leur soit exclusif, ces cérémonies sont constituées d'éléments tirés d'une pluralité de traditions, notamment non occidentales et préchrétiennes, et qui n'ont en commun que de représenter pour les participants les dépositaires d'une sensibilité plus authentique ou naturelle qui aurait disparu en Occident sous l'effet du patriarcat, du Christianisme, du capitalisme et de la technologie. Ainsi, on y retrouve bien des références explicites ou implicites aux Amérindiens (tambour, décorations, chansons et poèmes), à la mythologie grécoromaine (Diane, Aurora, Perséphone, des cheveux arrangés «comme faisaient les Grecs d'autrefois »), aux pratiques euro-américaines (bougies, gâteaux décorés) et latino-hispaniques (la quinceañera), et aux peuples germaniques et celtes (beaucoup d'aspects du cérémoniel wicca, mais aussi couronnes de fleurs, robes longues et flottantes et autres éléments issus d'une imagerie arthurienne popularisée par Le cycle $d^{\prime}$ Avalon de Marion Zimmer Bradley [1983]). Les adeptes des mouvements New Age et Néopaïen se voient souvent accusés soit de détourner des attributs de traditions culturelles qui ne sont pas les leurs sans respecter leur contexte d'origine, soit au 
contraire, de bricoler leurs pratiques à partir d'éléments hétéroclites issus d'un exotisme largement imaginaire. Mais de telles accusations, sans être fausses, se méprennent sur la raison de ces «emprunts » : ces éléments disparates sont mobilisés non pas en tant que modèles de comportement à réitérer, mais comme des ressources pour la construction d'événements qui se veulent à chaque fois originaux.

Nous touchons là à une autre caractéristique de ces rituels: ils se doivent d'être consciemment recomposés. Dans la quasi-totalité des autres traditions rituelles, les participants s'efforcent de répéter aussi fidèlement que possible des actes que d'autres auraient réalisé avant eux; une telle reconduction est conçue comme le garant même de l'efficacité du rite. Dans le cas des rites des premières règles, c'est au contraire l'innovation qui est de rigueur. Comme l'expliquent des intéressées elles-mêmes, et comme l'atteste la grande labilité des performances ainsi que les consultations auprès de la postulante, l'efficience de ces rituels requiert qu'ils soient élaborés et exécutés en fonction des circonstances et des individus concernés. En durcissant le trait, on peut dire que dans le cas des rituels de type canonique, l'inévitable introduction de modifications est subordonnée à l'intention de reproduire une même série d'actes, tandis qu'à l'inverse, dans ces rites contemporains, la récurrence effective de bon nombre d'actes cérémoniaux est subordonnée à un impératif de créativité.

En d'autres termes, à la différence de rituels de facture plus classique, et en corrélation avec l'attitude qu'ont les participantes envers les pratiques qu'elles empruntent à diverses traditions culturelles, les actions rituelles ne constituent pas une fin en soi mais un moyen. La nature exacte des actes que l'on accomplit au cours des rites des premières règles importe moins que ce que leur exécution permet de faire : fournir aux participants l'expérience d'une identité féminine originale reliant la postulante de manière nouvelle à la communauté des femmes. Si d'aventure telle ou telle conduite risquait d'empêcher les participantes d'éprouver cette réalité, on devra lui substituer une autre plus adéquate. Le mot d'ordre de ce type de ritualisation n'est donc pas de faire ce qu'il faut faire, mais de faire ce qu'on sent le mieux.

Ce que les personnes impliquées dans ces rites cherchent ainsi à reproduire ne sont donc pas les actions que d'Autres, estimés plus authentiques ou plus proches de la nature, auraient accompli antérieurement ou ailleurs, mais l'esprit dans lequel ils les auraient accomplies. Autrement dit, elles n'essayent pas de dupliquer un rite de puberté Navaho par exemple, mais d'être au plus près de ce qu'elles imaginent que des «Vielles sages » navaho feraient si elles étaient, comme elles-mêmes, des euroaméricaines de classe moyenne. Elles tentent plus exactement de redécouvrir les qualités affectives et intentionnelles qu'elles attribuent à des figures faisant autorité en matière de spiritualité, afin d'en faire la pierre de touche pour l'organisation des événements cérémoniels qu'elles entreprennent. En somme, à l'instar de nombreux rituels New Age et néopaïens, ces rites des premières règles s'organisent moins autour d'actions qu'autour de dispositions; ils se caractérisent non pas par des «actes archétypiques» [Humphrey et Laidlow, 1994] mais par des ressentis emblématiques.

$C^{\prime}$ est de cette façon qu'il convient de comprendre un aspect frappant et (pour un ethnologue) particulièrement dérangeant de ces rites contemporains qui les 
distingue radicalement des rituels de facture plus classique : la banalité flagrante des actions qui les composent. Non seulement ces actions, bien qu'inhabituelles, sont parfaitement intelligibles en termes de motivations et d'attitudes affectives quotidiennes, mais les participants ne cessent de convoquer des interprétations "symboliques » et des mises en parallèle métaphoriques afin d'en expliciter lourdement la signification. Ce travail de trivialisation des comportements rituels contraste nettement avec l'opacité soigneusement entretenue des activités rituelles propres à d'autres traditions, qui typiquement échappent à l'entendement ordinaire pour s'imposer aux participants comme autant de "pièges à penser » [Smith, 1973] hautement évocateurs mais singulièrement autoréférentiels.

Toutefois, la complexité qui fait si manifestement défaut sur le plan des actions, se retrouve sur le plan des agents. La participation à des pratiques ritualisées procède d'une présupposition pragmatique quelque peu différente de celle qui préside aux activités ordinaires: l'attention des acteurs porte moins sur la propension qu'auraient les dispositions des individus à s'exprimer au travers de leurs actes, que sur l'aptitude qu'aurait l'accomplissement de certains actes à induire chez eux des dispositions [Houseman, 2003]. Dans le cas d'activités rituelles de type canonique, la performance stipulée d'actes condensés et en partie inintelligibles dont la poursuite implique néanmoins l'expression émotionnelle et/ou intentionnelle, entraînerait chez les participants des états dispositionnels originaux (dont la nature exacte variera d'un individu à l'autre). Ce sont ces expériences, issues de la participation aux relations singulières actualisées au cours des rites, qui leur fournissent de nouveaux contextes à l'aune desquels ils peuvent réévaluer leur place dans le monde.

$C^{\prime}$ est également le cas chez les personnes qui participent à ces rites des premières règles: elles sont profondément et durablement affectées par les actes qu'elles exécutent. Mais les fondements de cette efficacité sont différents. Touchées par des actions qui procèdent non pas de leurs propres dispositions personnelles mais de celles d'Autrui qu'elles cherchent à retrouver en elles, les participantes agissent comme des sujets réfractés, partagés entre plusieurs identités différentes : les unes qui sont à l'origine des actes, les autres qui en ressentent les effets. L'indétermination qui signe la complexité propre à la participation rituel ne réside alors plus dans la question " que faisons-nous? ", mais dans une autre, plus intime : «qui sommes nous? ». Et cette dynamique de réfraction ne concerne pas seulement de vagues figures emblématiques (Vieilles sages amérindiennes, prêtresses celtiques, divinités gréco-romaines, etc.), mais se réalise aussi entre les participantes ellesmêmes qui sont continuellement en train d'infléchir leur comportement rituel en fonction de ce qu'elles ressentent que les autres participantes peuvent ressentir. Le résultat est la mise en place d'une réverbération intentionnelle et émotionnelle particulièrement intense où les participantes s'éprouvent (chacune à sa façon) comme étant plus qu'elles ne semblent être. Dotées d'identités féminines extraordinaires, multiples et amplifiées à la fois, elles s'imposent à elles-mêmes et aux autres comme des êtres exceptionnels, éminemment créatifs : des Déesses fécondes et fécondantes. 


\section{Références bibliographiques}

Biot Christian, 1993, La célébration des funérailles, Paris, Desclée de Brouwer.

Bradley Marion Zimmer, 1983, The Mists of Avalon, New York, Knopf.

Brown Judith, 1963, "A Cross-Cultural Study of Female Initiation Rites », American Anthropologist, new series 65 (4) : 837-853.

Diamant, Anna 1997, The Red Tent, New York, St Martin's Press. Trad.française : France-Loisirs, date

Dianteill, Erwan, Danielle Hervieu-Léger et Isabelle Saint-Martin (dir.), 2004, La modernité rituelle. Rites politiques et religieux des sociétés modernes, Paris, L'Harmattan.

Fedele Anna, 2008, Mary Magdalene. Menstrual Blood and Mother Earth, Thèse de doctorat, Ecole des Hautes Etudes en Sciences Sociales / Université Autonome de Barcelone.

Fellous Michèle, 2001, A la recherche de nouveaux rites, Paris, L'Harmattan.

Galinier Jacques et Anoinette Molinié, 2006, Les néo-indiens. Une religion du IIIe millénaire, Paris, Odile Jacob.

Grimes Ronald, 2002, Deeply into the Bone. Re-inventing Rites of Passage, Berkeley, University of California Press.

Handelman Don, 2004, Nationalism and the Israeli State: Bureaucratic Logic in Public Event, Oxford, Berg Publications.

Héritier Françoise, 1977, «L'identité samo » in L'identité. Séminaire dirigé par LéviStrauss, Paris, Grasset : 51-80.

Houseman Michael, 2003, «Vers un modèle anthropologique de la pratique psychothérapeutique », Thérapie Familiale 24 (3) : 289-312.

Houseman Michael, 2006, "Relationality », in Jens Kreinath, Jan Snoek et Michael Stausberg (dir.) Theorizing Rituals: Issues, Topics, Approaches, Concepts, Leiden, Brill Publications : 413-428.

Houseman, Michael, 2007, «Menstrual Slaps and First Blood Celebrations : Inference, Simulation and the Learning of Ritual », in David Berliner et Ramon Sarro (dir.) Learning Religion. Anthropological Approaches, New York, Berghahn Books, pp. 3148.

Humphrey, Caroline et James Laidlaw, 1994, The Archetypal Actions of Ritual. A Theory of Ritual Illustrated by the Jain Rite of Worship, Oxford, Clarendon Press.

Lassallette-Carassou Anne-Marie, 2008, Sorciers, sorcières et néopaïens dans l'Amérique d'aujourd'hui, Bordeaux, Presses Universitaires de Bordeaux.

Moisseeff, Marika, 2004, "Perspective anthropologique sur les rôles parentaux », in Pierre Angel et Philippe Mazet (dir.) Guérir les souffrances familiales. 50 spécialistes répondent, Paris, Presses Universitaires de France.

Owen Lara, 1993, Her Blood is Gold, San Francisco/Thorsons, Harper/Aquarian. Trad française Son s ng est d'or..

Salomonsen Jone, 2002, Enchanted Feminism. Ritual, gender and Divinity among the Reclaiming Witches of San Francisco, London/New York, Routledge.

Segalen Martine, 2003, Eloge du mariage, Paris, Gallimard.

Smith Pierre, 1979, "Aspects de l'organisation des rites », in Michel Izard et Pierre Smith (dir.), La fonction symbolique, Paris, Gallimard : 139-170. 
Soster-Olmer Ksenija, 2001, "First moon rising: the making of a menarche ritual », Mothering 109 (enregistré 03/2006 à http://www.mothering.com/community tools/teen_voices/first-moon.html.

\section{RÉSUMÉ}

Cet article propose une description et une analyse des rituels célébrés lors des premières menstruations dans l'Occident contemporain. À la différence des initiations féminines exécutées dans d'autres sociétés, ces rites de passage ne médiatisent ni l'accession de la postulante au statut d'adulte, ni une reconnaissance formelle de ses aptitudes à procréer. Ils lui fournissent l'expérience d'une identité féminine originale, celle d'un être spirituel doté de capacités créatives, la consacrant ainsi comme agent indépendant au sein d'une communauté de femmes vitales et responsables.

Mot-cles : Rituel. Menstruation. Néopaïenisme. Initiation. Femmes

\section{ABSTRACT}

First menstruation rituals in contemporary Western society

This article describes and analyses rituals celebrating a young woman's first menstruation in contemporary Western society. Unlike feminine initiations in other societies, these rites of passage neither mediate the young woman's access to adult status, nor do they consist in a formal recognition of her ability to bear children. In providing her with the experience of an enhanced feminine identity, that of a creative, spiritual being, they establish her as an independent agent within a community of vital and responsible women.

Key words: Ritual. Menstruation. Neopaganism. Initiation.Women.

Michael Houseman

Centre d'Etudes des Mondes Africains (CEMAf)

27 rue Paul Bert

94202 Ivry-sur-Seine

houseman@attglobal.net 